\title{
Estimación de la talla, adaptando la técnica de medición altura talón-rodilla con regla y escuadra
}

\author{
Adapting the Heigth Heel-Knee measure technique \\ with Ruler and Square, Estimation of the Height
}

Mtra. María de Lourdes Ildeliza Sierra Torrescano*

*Profesor de carrera Asociado B, ENEO UNAM

\section{Resumen}

Introducción: La talla o estatura es uno de los indicadores antropométricos que se utiliza para la valoración del estado nutricional, con mayor frecuencia.

La medición de la estatura de los adultos mayores a través de la técnica de mayor exactitud en bipedestación o de pie, en algunos casos es difícil de determinar, por ello la utilización de otro segmento del cuerpo para estimarla como es la altura talón rodilla, puede ser muy útil para obtener el Índice de Masa Corporal (IMC).

El instrumento para medir el largo de pierna es un antropómetro, el cual es costoso y poco accesible para el equipo de salud, en general.

Objetivo: Estimar la talla en adultos mayores, adaptando la técnica de medición altura talón-rodilla con regla y escuadra

Metodología: Se realizó un estudio exploratorio en una muestra de 59 adultos mayores, de una comunidad suburbana, a quienes se les aplicó una encuesta, en donde se registró la edad como criterio de inclusión e indicador para la estimación de la talla. Se realizó la antropometría mínima que consistió en primer término, en la medición de la talla, determinada con estadiómetro y con la altura talón-rodilla, técnica adaptada con regla y escuadra. La talla se estimó utilizando las ecuaciones desarrolladas por Chumlea, (1985). La medición del peso se obtuvo a través de una báscula portátil; con base en el resultado de peso y talla se calculó el Índice de Masa Corporal (IMC).

Resultados: De los 59 adultos mayores 42 fueron mujeres y 17 hombres, la media de edad fue de 72 años y el límite máximo fue de 99 años.
En cuanto a los rangos de peso encontrados van desde $36.9 \mathrm{Kg}$. como límite inferior hasta $84 \mathrm{Kg}$. como límite máximo; la media encontrada fue de $62.6 \mathrm{Kg}$. y la mayor frecuencia de 3 individuos la encontramos en $63 \mathrm{Kg}$.

Se correlacionó la talla real versus la talla estimada, encontrando significancia estadística de $(p<=0.0002)$, sin embargo es más significativa la correlación en hombres $(r$ $=0.8591$ ).

Se logró adaptar la técnica de medición de la altura talón-rodilla con una regla y escuadra, lo que permitió facilitar la estimación de la talla, por lo accesible y económico de los instrumentos.

Discusión: La estimación de la talla de los adultos mayores a partir de la altura talón-rodilla con regla y escuadra, demostró su precisión al correlacionarla estadísticamente, ya que se obtuvo una $r$ de 0.8591 semejante a lo reportado por Huey-Shinn y cols. y Hernández y cols.

Lo anterior sustenta que la estimación de la talla en adultos mayores a través de la técnica adaptada de medición altura talón-rodilla con regla y escuadra es un procedimiento antropométrico alternativo para realizar la evaluación nutricional y establecer diagnósticos de estado nutricio en adultos mayores, a su vez también es una opción procedimental para la práctica del profesional de Enfermería que con mayor frecuencia realiza estas prácticas en primer nivel de atención.

Palabras clave: altura talón-rodilla, antropometría, evaluación del estado nutricional y enfermería. 


\section{Abstract}

The nutricional assessment require of the height, which is the more frecuency anthropometric indicator used.

In some cases the major adult's height is difficult to determine, that's why the estimation using other body's segment as height heel-knee might be very useful for the nurse staff.

Objetive: To estimate the major adult's height using the measurement of the height heel-knee with ruler and square versus the real height measurement with stadiometer.

Metodology: A descriptive exploratory study was realized in a mayor adult sample, of a suburban community, to wich them were inquiryied the age was registered, that is indicate that it'll be serving of inclusion criterion as part of height estimation data.

There were realized the weight minimal anthropometric, stadiometer height determinity and it was adapted with ruler and square technique, the height heel-knee, the weight was obtained to have the Body Mass Index (BMI).
The height has estimated using Chumlea's (1985) unfolding equations.

Results: Of 59 mayor adults, 42 were women and 17 were men, in age relation, the mean was 72 years, that's indicate in question about relativing "young" mayer adults population; accord of Geriatry criterion and 99 years at the most limit.

In regard to weight ranges found, there is form $36.9 \mathrm{Kg}$ as minimum limit to $84 \mathrm{Kg}$. as the most limit; the mean found was $62.6 \mathrm{Kg}$ and the most frecuency was $63 \mathrm{Kg}$. in 3 individuals.

The technique of measurement with a rule and square adapted and it was found more stadistic correlation in the height estimation using the heel-knee in men, using the Chumlea equations.

Key words: height heel-knee, anthropometry, nutritional assessment and nursery

\section{INTRODUCCIÓN}

La antropometría es una herramienta muy útil para el personal de enfermería, en el área clínica, sin embargo a veces se limita su utilidad debido a la falta de confiabilidad de las mediciones, por ello es necesario que el profesional de enfermería se capacite en este campo de estudio.

Los indicadores antropométricos son métodos prácticos con los que se obtiene una evaluación general de los cambios en las dimensiones corporales, relacionados con la edad, así como su influencia en el estado de nutrición y el riesgo de morbilidad y mortalidad. Alemán ${ }^{1}$

La talla o estatura es uno de los indicadores antropométricos que con mayor frecuencia se utiliza para la valoración del estado nutricional, siendo importante para desarrollar índices como la relación peso/talla, IMC, índice creatinina-altura, así como en ecuaciones para estimar el gasto energético basal en adultos. Por ello, si no es posible determinar la altura corporal, estos índices no podrían calcularse, perdiendo una valiosa información. Dudet ${ }^{2}$

La técnica para realizar la medición es relativamente sencilla; sin embargo, se requiere de rigor metodológico para obtener la precisión, exactitud y por consiguiente la confiabilidad para establecer diagnósticos, que tienen gran utilidad en la prevención de enfermedades para el profesional de enfermería.
La medición de la talla puede presentar dificultades en los adultos mayores que no pueden permanecer de pie y mantener una postura erecta, que presenten alteraciones físicas en la columna vertebral, o se encuentran hospitalizados con ciertas condiciones clínicas que limitan la ejecución adecuada de la técnica. Considerando éstas y otras variables algunos investigadores han utilizado otros segmentos corporales como: la brazada, la media brazada y la altura talón-rodilla, las cuales han demostrado buena correlación con la talla real, como lo reportan Hernández y col. ${ }^{3}$

La brazada es la distancia que existe entre la punta de los dedos (el más largo) de la mano derecha, y las puntas de los dedos (el más largo) de la mano izquierda, (sin contar las uñas) cuando los brazos se encuentran extendidos lateralmente a la altura de los hombros. Tapia ${ }^{4}$

La aplicación de la técnica de la brazada requiere que los miembros superiores estén totalmente extendidos, lo que para muchos pacientes hospitalizados representa una limitante, cuando se encuentran rodeados de aparatos que reducen el espacio para tomar la medición, o no cuenten con la capacidad física para lograr dicha extensión.

La media brazada es la medición del centro de la escotadura esternal a la punta del dedo medio izquierdo o derecho según la fuente de comparación. Vargas ${ }^{5}$. 
El estudio de la media brazada según Hernández y col. ${ }^{6}$ mostró un comportamiento muy parecido a la brazada para el sexo masculino; sin embargo, en las mujeres su asociación con la talla obtuvo valores más bajos.

Chumlea estimó la talla a partir de la altura talón rodiIla, (con base en el estudio de que los huesos largos no sufren modificaciones con la edad) y desarrolló las ecuaciones para obtenerla, en varios estudios se ha demostrado su precisión en la estimación de la talla, como lo reportan Hernández y col. ${ }^{6}$, quienes además confirmaron mayor precisión de esta medición en los hombres que en las mujeres. Resultados similares fueron reportados por Huey-Shinn y col. Citado en ${ }^{6}$, en un grupo de ancianos de Taiwán, en quienes observaron mayores valores de correlación de altura de rodilla con la talla para el sexo masculino.

El instrumento para medir la altura talón rodilla es un antropómetro, el cual tiene un elevado costo y no es común que exista en la mayoría de las clínicas y hospitales.
La talla presenta ciertas dificultades para tomarla en algunos pacientes geriátricos, por lo que el objetivo de este estudio fue contar con una adaptación de la medición de la altura talón rodilla, con un instrumento de medición más sencillo, económico y de fácil manejo, como es una regla en forma de "L" que se conoce comúnmente como regla "T" de dibujo semiprofesional y una escuadra tipo escolar para cuidar el ángulo de $90^{\circ}$, de manera muy similar a como aparece en la siguiente figura 2.

Como se muestra en la figura se adaptó el instrumento de medición pero se conservó el rigor metodológico en la toma de la medición.

El personal de enfermería puede capacitarse en la toma de esta medición y lograr estimar la talla de los adultos mayores que presenten limitaciones físicas que no les permitan mantener una postura erecta.

Por otro lado, la participación de enfermería en la detección oportuna de sobrepeso y obesidad calcula-

\section{Figura 2.}

Adaptación de la técnica de medición de la altura talón rodilla con regla y escuadra.

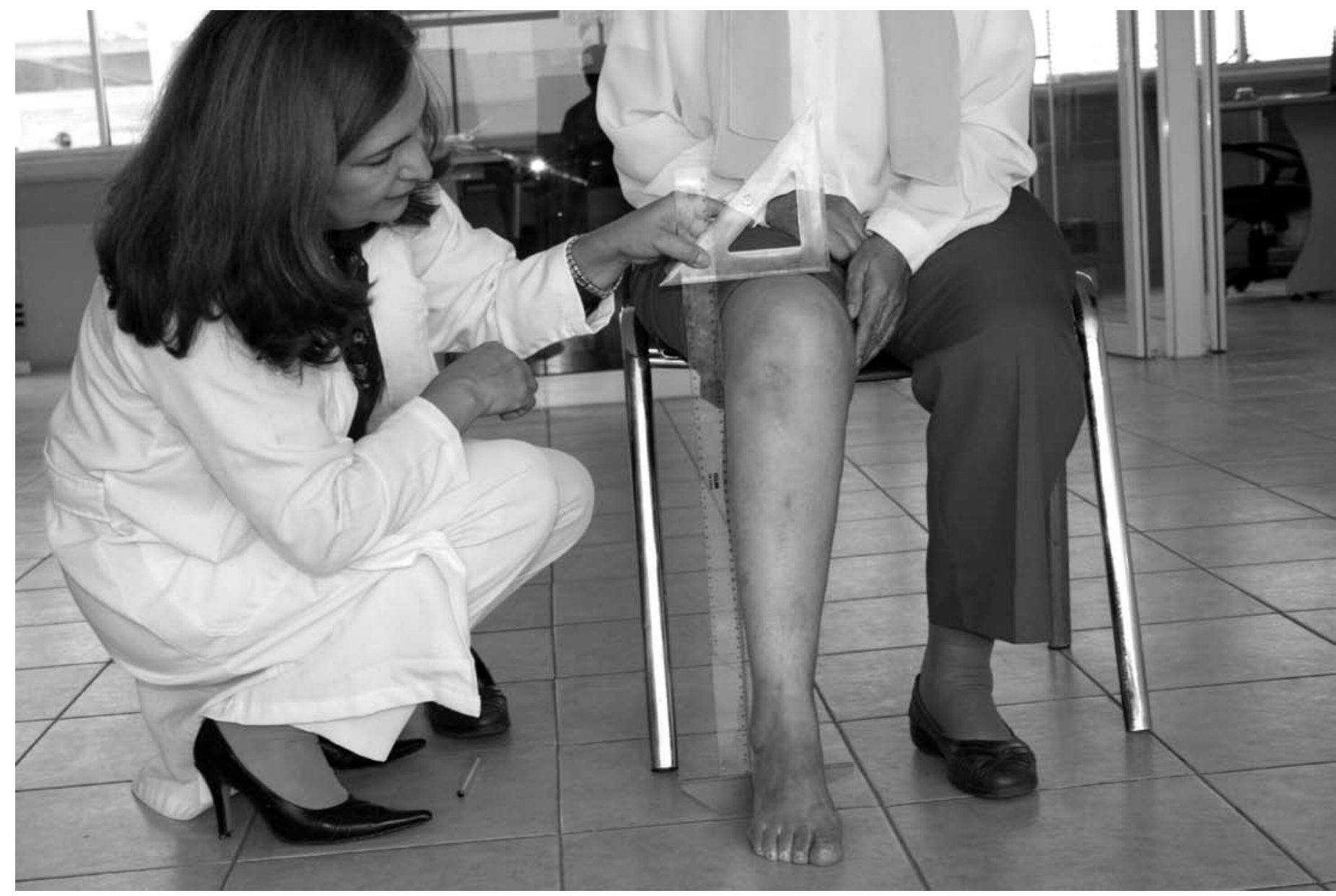


da con el Índice de Masa Corporal a través de la relación del Peso y la Talla en adultos mayores es de suma importancia dado el papel protagónico que tiene este profesional en la prevención de enfermedades crónico degenerativas.

Existen otros reportes de estudios de la altura talón rodilla medida con cinta métrica, Guzmán, y col. ${ }^{7} 2005$, sin embargo, se requirieron hacer ajustes a las ecuaciones para determinarla por sexo.

\section{OBJETIVO}

Estimar la talla de los adultos mayores utilizando la medición de la altura talón rodilla con una regla y escuadra y confrontarla con la talla real medida con estadiómetro.

\section{MATERIAL Y MÉTODOS}

Se realizó un estudio exploratorio - descriptivo en una comunidad suburbana, perteneciente a la Delegación de Xochimilco, Distrito Federal. La comunidad cuenta con un Centro Comunitario de Atención Primaria a la Salud (CCOAPS) de la Escuela Nacional de Enfermería y Obstetricia de la Universidad Nacional Autónoma de México (ENEO-UNAM), el cual es atendido por pasantes de la Licenciatura en Enfermería y Obstetricia.

Se utilizó un diseño de campo tipo encuesta y se trabajó con una muestra. El tamaño de la muestra se calculó con el paquete Epi Info versión 6 en el módulo Statcalc con un $90 \%$ de confiabilidad para una población estimada de 650 adultos mayores residentes de la comunidad. El tamaño de muestra mínimo fue de 59 personas. El CCOAPS ha dividido geográficamente a la comunidad en cuatro módulos A, B, C y D para facilitar su estudio, el marco muestral fue no probabilístico y se realizó con representación de los cuatro módulos.

El criterio de inclusión fue adultos mayores de 60 años y más, concientes, que pudieran deambular, residentes de la comunidad y que aceptaran participar en el estudio firmando o poniendo una cruz en la carta de consentimiento informado, donde se explicó el carácter voluntario de su participación y los procedimientos a realizar. En el estudio se incluyeron adultos mayores de uno y otro sexo. Los criterios de exclusión fueron adultos menores de 60 años, no residentes de la comunidad, con deformaciones de la columna vertebral, con incapacidad para ponerse de pie por sí solos y no ambulatorios. A cada uno de los participantes se les aplicó una encuesta con datos generales y se les realizaron las mediciones antropométricas.
Los datos se registraron el mismo día que se llevaron a cabo y por el mismo observador.

\section{Mediciones antropométricas}

Las mediciones se tomaron en la casa de los adultos mayores, por lo que se utilizaron básculas y estadiómetros portátiles.

El peso corporal se registró en kilogramos, colocando al adulto mayor de pie en una báscula portátil, sin zapatos, con la ropa mínima que permitiera quitarse, en el centro de la báscula y sin objetos que pudieran modificar la medición.

La talla se obtuvo en centímetros, se usó un estadiómetro portátil de madera, el sujeto se colocó sin zapatos, en posición erecta, hombros relajados, brazos a los costados, la cabeza libre de accesorios o peinados que alteren la medición, se cuidó la posición de la cabeza, (plano de Frankfort), los talones juntos y las puntas de los pies ligeramente separados, debiendo estar en contacto con un plano vertical.

El plano de Frankfort $^{8}$ se ejemplifica con la siguiente figura 3. y se define como:

La línea imaginaria que va desde el agujero infraorbitario al conducto auditivo externo, paralela al piso y con la vista al frente, lo que permite tomar la medición correcta.

\section{FIGURA 3}

\section{Fotografía de la técnica de medición de la estatura verificando el Plano de Frankfort}

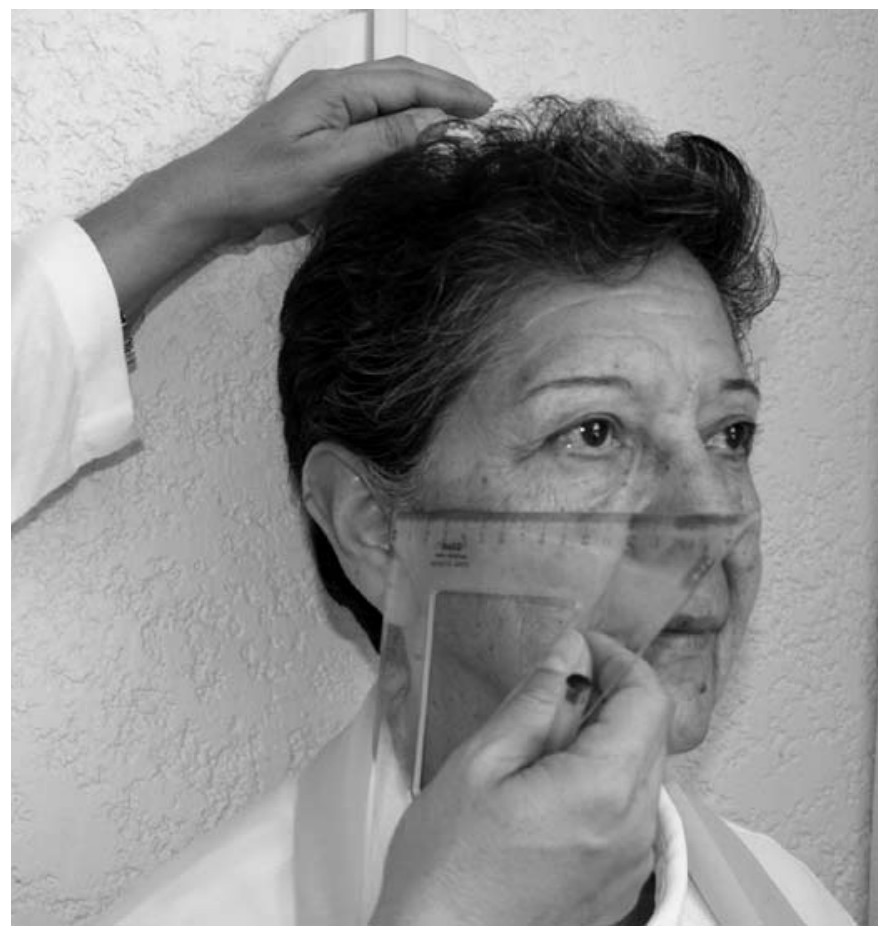


Técnica de medición de la altura talón rodilla. El sujeto debe estar en posición sedente, sin zapatos, flexionando la rodilla y tobillo en ángulo de $90^{\circ}$, se mide la longitud de la pierna colocando la regla bajo el talón y situar el eje de tal modo que pase por el maleolo externo justo por detrás de la cabeza del peroné. Se desliza la escuadra sobre la superficie anterior del muslo. Por encima de los cóndilos del fémur, a unos $4 \mathrm{~cm}$. de la rótula. Se sostiene el eje de la regla de manera paralela al eje de la tibia y se presiona para comprimir tejidos, para tomar la longitud en centímetros, como se observa en la figura 2.

Para corroborar la utilidad de esta medición con la adaptación del instrumento de medición de la altura talón rodilla se confrontaron las mediciones de la talla real medida con estadiómetro versus la altura talón rodilla utilizando las siguientes ecuaciones descritas por Chumlea ${ }^{9}$ para la predicción de la talla:

Talla hombres $(\mathrm{cm})=\{2.03 \times$ altura-rodilla $(\mathrm{cm})\}-\{$ $0.04 \times$ edad (años) $\}+64.19$

Talla mujeres $(\mathrm{cm})=\{1.83 \times$ altura-rodilla $(\mathrm{cm})\}-\{$ $0.24 \times$ edad (años) $\}+84.88$

En estas ecuaciones se observa que la edad se utiliza como indicador para la estimación de la estatura.

Con los datos de peso y talla se calcula el IMC = Peso / (Talla) ${ }^{2}$, éste índice también conocido como Quetelet es uno de los indicadores más empleados para medir el estado de nutrición en la población adulta; una de sus ventajas es que no requiere de tablas de referencia.

Los puntos de corte para el IMC según Casanueva, y col. ${ }^{10}$ en los que se considera hombre y mujer y coinciden con los utilizados por Velázquez. C en diversos estudios, éstos son:

$\begin{array}{ll}\text { Sobrepeso } & \mathrm{IMC}>27.8 \text { Hombre } \\ \text { Sobrepeso } & \mathrm{IMC}>27.3 \text { Mujer } \\ \text { Obesidad } & \mathrm{IMC}=0 \text { O }>30\end{array}$

Para el análisis estadístico se realizó una base de datos y se utilizó el software estadístico SPSS Versión 9, obteniendo porcentajes y la correlación estadística.

\section{RESULTADOS}

Se incluyeron 59 adultos mayores: 42 mujeres (71\%) y 17 hombres (29\%) sin que esto concluya que haya en esa proporción mayor cantidad de mujeres en la comunidad, ya que por el horario en el que fue aplicada la encuesta en sus domicilios corresponde al tiempo de jornada laboral para muchos adultos mayores masculinos que aún trabajan.

En relación a la edad, el límite inferior fue de 60 años de acuerdo al criterio de inclusión, la mayor frecuencia de 5 individuos se encontró en esta edad. La media fue de 72 años y el límite máximo fue de 99 años.

En cuanto a los rangos de peso encontrados van desde $36.9 \mathrm{Kg}$. como límite inferior hasta $84 \mathrm{Kg}$. como límite máximo; la media encontrada fue de $62.6 \mathrm{Kg}$. y la mayor frecuencia de 3 individuos la encontramos en $63 \mathrm{Kg}$.

De los datos de talla se encontró una estatura de 1.32 m. como límite inferior, la media fue de $1.49 \mathrm{~m}$. y el límite máximo fue de $1.75 \mathrm{~m}$.

El IMC se calculó con los puntos de corte de Casanueva y col. y se obtuvo lo siguiente:

CUADRO No. 1

Normalidad, Sobrepeso y Obesidad en Mujeres Adultas Mayores de la Comunidad Suburbana

\begin{tabular}{|l|c|c|}
\hline & Frecuencia & Porcentaje \\
\hline Normalidad & 16 & 27.1 \\
\hline Sobrepeso & 10 & 16.9 \\
\hline Obesidad & 16 & 27.1 \\
\hline Total & 42 & 71.2 \\
\hline
\end{tabular}

Se detectó un $38.1 \%$ de normalidad, un $23.8 \%$ de sobrepeso y un $38.1 \%$ de obesidad, en mujeres.

CUADRO No. 2

Normalidad, Sobrepeso y Obesidad en Hombres Adultos Mayores de la Comunidad Suburbana

\begin{tabular}{|l|c|c|}
\hline & Frecuencia & Porcentaje \\
\hline Normalidad & 11 & 64.7 \\
\hline Sobrepeso & 5 & 29.4 \\
\hline Obesidad & 1 & 5.9 \\
\hline Total & 17 & 100 \\
\hline
\end{tabular}


Para los hombres encontramos un $64.7 \%$ en la normalidad, un $29.4 \%$ de sobrepeso y sólo un $5.9 \%$ de obesidad.

Con estos datos se corrobora la utilidad de la medición de la estatura para determinar diagnósticos del estado nutricional a través del cálculo del IMC.

En cuanto a los datos de altura talón-rodilla que presentaron los adultos mayores se encontró el límite inferior de $42.5 \mathrm{~cm}$. hasta $52 \mathrm{~cm}$. como límite máximo que corresponde al señor de mayor estatura.

La correlación entre la talla real y la calculada por las ecuaciones es para la mujer de:

$$
r=0.6285 \text { ( } p<=0.0002 \text { ) (Ver fig. 4). }
$$

Este valor de $r$ es la correlación e indica que existe una relación estadísticamente muy significativa entre las dos mediciones de la talla, entre más cercana a 1 > es la asociación entre las dos variables involucradas en su cálculo.

Cuando la p asociada a un coeficiente de correlación es $>0.05$, se dice que no hay una asociación entre las dos variables que se están correlacionando.

Por otra parte, cuando la p asociada a este coeficiente de correlación es muy pequeña, se dice entonces que existe una gran fuerza de asociación entre las dos variables de donde se calculó dicho coeficiente.

En cuanto al hombre los resultados fueron:

$$
r=0.8591(p<0.0002)(\text { Ver fig. 5) }
$$

El valor de r es más significativo en el hombre, ya que es mayor este valor que en el de la mujer.

\section{FIGURA 4}

Talla real de las mujeres versus talla calculada altura talón - rodilla

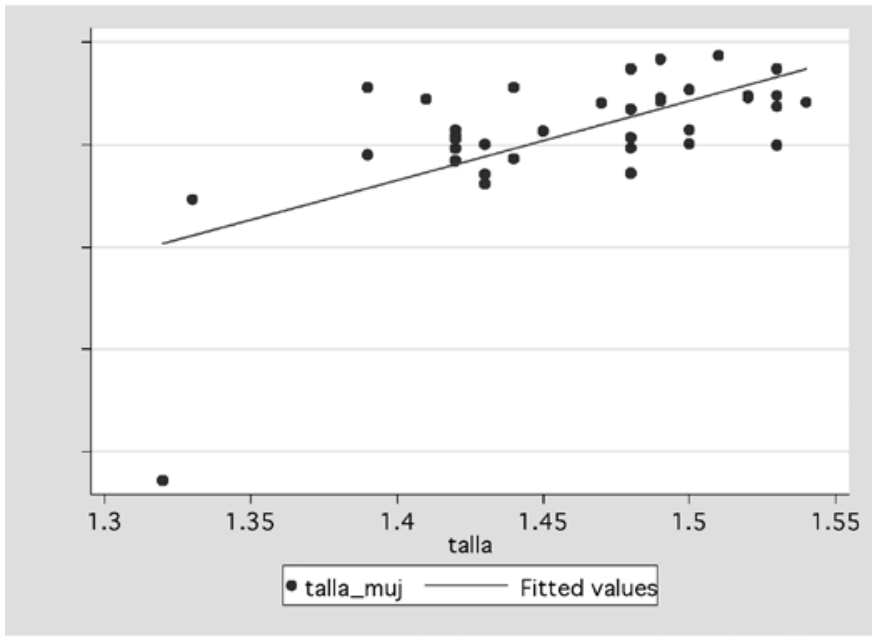

En esta figura, se observan menos de 42 mujeres, la razón es que algunas de las tallas son muy similares o iguales a las de otras y el "punto" en la gráfica se empalma con el de una persona de similar o igual talla.

\section{Figura 5 Talla real de los hombres versus talla calculada altura talón - rodilla}

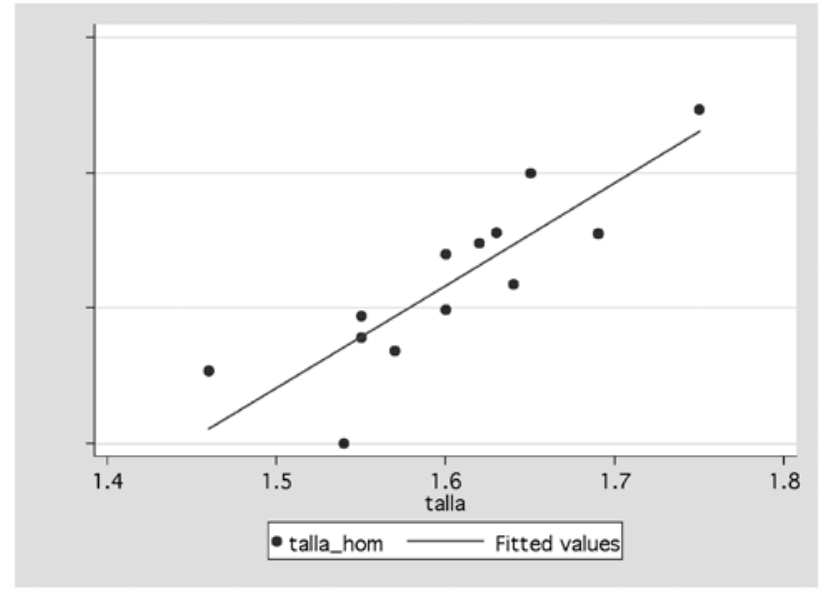

Se graficaron los resultados encontrando significancia estadística tanto en hombres como en mujeres.

Se encontró mayor correlación estadística en la estimación de la estatura utilizando la medición de la altura talón rodilla en los hombres, que coincide con lo reportado por otros autores, como en el estudio de Guzmán-Hernández y col., en otro estudio reportado por Huey-Shinn y col en un grupo de ancianos en Taiwán. En otro estudio Bermudez y col. reportaron valores más altos de correlación. También en el estudio reportado por Hernández- Hernández y col., se evidenció que la altura de rodilla mostró la mayor correlación con la talla.

\section{DISCUSIÓN}

Los avances en la ciencia y tecnología obligan a la disciplina de enfermería a actualizarse en los diferentes campos de estudio, la antropometría es una herramienta muy útil, en el área clínica, sin embargo, se requiere seguir procedimientos estandarizados, lo que incrementará la precisión y exactitud de las mediciones.

La estatura es uno de los indicadores antropométricos que con mayor frecuencia se utiliza para la valoración del estado nutricional, al relacionarlo con el peso y obteniendo así el IMC, se detectó un 38.1\% de normalidad, un $23.8 \%$ de sobrepeso y un $38.1 \%$ de obesidad, en mujeres, 
para los hombres se encontró un $64.7 \%$ en la normalidad, un $29.4 \%$ de sobrepeso y sólo un $5.9 \%$ de obesidad.

Al obtener el IMC se confirma la utilidad de la medición de la estatura para determinar diagnósticos del estado nutricional, de manera rápida y sencilla.

La medición de la altura talón rodilla es una buena alternativa para predecir la talla de los adultos mayores, que por sus limitaciones físicas no pueden tomar una postura erecta. La adaptación de esta técnica de medición con regla y escuadra, logró una buena correlación con mayor significancia estadística en los hombres, con un valor de $r=0.8591$, lo que coincide con lo reportado por otros autores, como en el estudio de Guzmán Hernández y col. que encontró la mayor correlación con la talla en el grupo total de los hombres $r=0.82$ y en Huey-Shinn y col en un grupo de ancianos en Taiwán, encontraron valores de correlación $r=0.77$, para el sexo masculino y en otro estudio Bermudez y col. reportaron valores más altos de correlación en ancianos hispanos $r=0.84$ para los hombres y ligeramente más bajos para las mujeres, $r=0.82$. También en el estudio reportado por Hernández- Hernández y col., se evidenció que la altura de rodilla mostró la mayor correlación con la talla en el grupo total de los hombres $(r=0,82)$ y en los de 60-79 años $(r=0,84)$. Para el sexo femenino, esta variable obtuvo correlaciones más bajas con la talla y especialmente para el grupo de 80 años y más $(r=0,59)$.

La adaptación de la técnica de medición de la altura talón rodilla con regla y escuadra, demostró confiablidad estadística con valores de $\mathrm{p}<0.0002$ al correlacionarla con la talla real medida con la técnica de mayor exactitud que es en bipedestación o de pie, además los instrumentos utilizados son de muy bajo costo y accesibles a todo el personal de enfermería, lo que permite recomendarla como una medición fácil y accesible.

La altura talón-rodilla es un procedimiento antropométrico alternativo, para realizar la evaluación nutricional y establecer diagnósticos de estado nutricio en adultos mayores, a su vez, también es una opción procedimental para la práctica del profesional de Enfermería que con mayor frecuencia realiza estas prácticas en primer nivel de atención.

\section{AGRADECIMIENTOS}

Se agradece al joven Diego Olascoaga Alba, su valiosa participación en la realización del material fotográfico de este documento.

\section{REFERENCIAS BIBLIOGRÁFICAS}

1 Alemán-Mateo, H., y col. Los indicadores del estado de nutrición y el proceso de envejecimiento. Nutrición Clínica. 2003; 6 (1): 46-52.

2 Dudet, M. E. Estimación de la altura corporal en población española menor de 60 años. Rev. Esp. Nutr Comunitaria 2004; 10(2):74-81.

3 Hernández, R. y col. Selección del predictor más adecuado para estimar la contextura en un grupo de adultos mayores institucionalizados y de vida libre en Venezuela. ALAN. 2003: 53 (3): 251-257.

4 Tapia, J. y col. Parámetros objetivos regionales de evaluación nutricional en una población de adulto mayor. Nutrición Clínica. 2003; 6 (1): 27-35.

5 Vargas, L. Fundamentos para la evaluación antropométrica del estado de nutrición de los ancianos. Cuadernos de Nutrición. 1997; 20 (2): 6-13.

6 Hernández, R. y col. Segmentos corporales y talla en un grupo de adultos mayores venezolanos. Invest. Clín. 2005; 46 (3).

7 Guzman-Hernández, C., Reinoza-Calderon, G. y Hernández-Hernández, R. A. Estimación de la estatura a partir de la longitud de pierna medida con cinta métrica, Nutr. Hosp. [online]. 2005, vol. 20, no. 5 [citado 2008-07-11], pp. 358-363. Disponible en: <http://scielo.isciii.es/scielo. php?script=sci_arttext\&pid=S0212-16112005000700 009\&lng =es\&nrm =iso $>$. ISSN 0212-1611

8 Mazza, Juan C. Mediciones antropométricas. Estandarización de las técnicas de medición, actualizada según parámetros internacionales. PubliCE Standard. 27/10/2003. Pid: 197.

9 Espinosa-Cuevas, M. A., Velázquez-Alba, M. C. La Composición Corporal y su utilidad en el diagnóstico del Estado de Nutrición. Aplicación Clínica en Adultos. Libro de Trabajo. Sociedad de Nutriología, A. C, México, 2001.

10 Casanueva, E. et. Al. Nutriología Médica. Editorial Médica Panamericana, México, 1995.

\section{DIRECCIÓN PARA CORRESPONDENCIA}

Mtra. María de Lourdes Ildeliza Sierra Torrescano

Email:mlildesierra@hotmail.com 